\title{
¿De dónde proviene el financiamiento de las PyMES?
}

\author{
Kareeme Davila Cisneros ${ }^{1}$ \\ ${ }^{I}$ Estudiante de la Maestría en Dirección y Gestión de Negocios Internaciones, Facultad de Contaduría \\ Pública y Administración, kareeemedavila15@gmail.com, Niños héroes, Ciudad Universitaria, San Nicolás \\ de los Garza, N.L., 8113404430
}

Información del artículo revisado por pares

Fecha de aceptación: junio-2021

Fecha de publicación en línea: diciembre-2021

DOI: https://doi.org/10.29105/vtga7.1-113

\section{Resumen}

Las MIPyMES son un parte fundamental para la economía mexicana dado a las implicaciones que de ellas se desglosan. Estas empresas enfrentan muchos desafíos, la mayoría cuando inician operaciones. Dentro de esos desafíos, está el acceso a un financiamiento con algún banco u otras instituciones financieras privadas. Dado a esta necesidad de recursos, estas empresas recurren a otro tipo de entidades para adquirir los recursos que necesiten para el fin deseado, ya sea la inversión en nuevos proyectos, liquidación de deuda o en si la sobrevivencia del negocio. Con esta afirmación, nace la duda de donde proviene el financiamiento de las MIPyMES en México. Gracias a la Encuesta Nacional de Micronegocios (ENAMIN, 2012) se puede conocer las fuentes que utilizan los mexicanos para obtener sus recursos. Este estudio pretende hacer conciencia de la importancia de estas empresas, asimismo como la importancia de que sigan operando, evitar bancarrotas y moviendo la economía mexicana.

Palabras clave: banco, otras instituciones financieras privadas deuda

\section{INTRODUCCIÓN}

Las empresas que son consideradas como micronegocios, pequeñas y medianas se conocen como MIPyMES, las cuales son de gran importancia para la economía mundial dado que representan un gran porcentaje del
Abstract
The SME's are fundamental part of the Mexican economy given the implications that this companies have. This companies fase a lot of challengues, and most of them are when they start. On those challengues, the access to a loan with a bank. Given the need of those resources, this companies need to search other non-bank financial institution to get those resources to accomplish their objective, this could be an investment, pay for a loan or even the survival of the bussiness. With this afirmation, the doubt of where the loans come from for mexican SME's arises. Thanks to the National Survey of SME's (ENAMIN 2012) the sources that mexicans use to finance themselfs can be studied. This study pretends to make awareness of the importance of this SME's given the importance of them to continue operating, avoid banktrupcy and moving the mexican economy.

Keywords: bank, non-bank financial institution, bankruptcy

JEL: G21, G23, G33

comercio global. Romero (2006) señala que "en la mayor parte de los países de la OCDE (Organización para la Cooperación y el Desarrollo Económico), las MIPyMES representan entre $96 \%$ y $99 \%$ del número total de empresas y aportan aproximadamente una sexta parte de la producción industrial". En 
México, estas empresas conforman un gran porcentaje y son de suma importancia debido a sus contribuciones en la economía mexicana. Dado a diversas limitaciones, las MIPyMES afrontan más retos que una empresa grande e internacional. Estos retos representan un factor de alto impacto en su esperanza de vida. Luna et al. (2016) establecen que de cada cien MIPyMES que inician actividades, después de un año sobreviven solo 24, a los cinco años sobreviven y a los 25 años sobreviven solo nueve de ellas.

Dentro de estos retos se pueden incluir el crecimiento, la innovación, el acceso a un financiamiento, entre otros. El factor del financiamiento es de suma importancia para estas empresas dado que muchas veces es necesario para lograr un crecimiento y avance. El tema nace cuando estos financiamientos son negados o no son otorgados a los candidatos de las MIPyMES que aplican a uno.

Con esta tesina se pretende conocer cuáles son los métodos de financiamiento para las MIPyMES en México, con base en la Encuesta Nacional de Micronegocios (ENAMIN) conducida en el año 2012. En el presente trabajo se realizará un análisis de la procedencia del financiamiento de las MIPyMES.

En este documento se desarrollarán cinco capítulos que comprenderán lo siguiente: el primero describirá el marco contextual del estudio, continuando con el segundo capítulo en el que se hará referencia al marco teórico en el que se podrán ver temas relacionados con el financiamiento a MIPyMES en cuanto a su historia, importancia, finalidad, así como los factores involucrados para que se pueda obtener el financiamiento, hasta concluir con un enfoque teórico. En el tercer capítulo se explicará el método utilizado en esta tesina. En el cuarto capítulo se muestran los hallazgos de la aplicación de la metodología de la investigación y los resultados obtenidos. Por último, en el quinto capítulo se exponen las conclusiones y recomendaciones de la tesina.

\section{MARCO TEÓRICO}

Para comenzar con el estudio acerca del financiamiento de las MIPyMES en México, haremos una revisión de la literatura de este tema en específico

\subsection{Antecedentes teoricos}

Las MIPyMES en México son de suma importancia para diversos actores en el país; es importante mencionar que para el año 2019 representaban un total de 4.1 millones de empresas en México (INEGI, 2019). Por esta razón, estas empresas abarcan gran parte del Producto Interno Bruto (PIB) mexicano y contribuyen de manera exponencial a las tasas de empleo. Según la Organización para la Cooperación y el Desarrollo Económicos (OCDE, 2018) "las MIPyMES desempeñan un papel fundamental en el crecimiento económico, la creación de empleo, el desarrollo local, regional y la cohesión social". Nos comentan los autores Alvarado, Ortiz y Morales (2018) que estas empresas representan la columna vertebral de la economía nacional debido a los acuerdos comerciales y al apoyo que reciben los emprendedores en sus negocios o proyectos que son muy productivos.

Al ser empresas, en su mayoría micronegocios, recién formadas, con un número bajo de empleados, con poco acceso a la reinversión o que carecen de activos que proporcionar como aval, las MIPyMES afrontan grandes retos en muchos aspectos. Estos pueden ser aspectos financieros, de competencia, operativos, etcétera.

Uno de los aspectos más importantes dentro del ámbito financiero es el acceso a un financiamiento. Estos financiamientos pueden ser solicitados con una entidad bancaria, una SOFOM, con familiares, entre otros. Según López (2018) "hay PyMEs que una de sus principales preocupaciones es obtener los recursos necesarios para su productividad y sobrevivencia". Cuando una empresa tiene el capital o recursos suficientes para subsistir los primero meses o años, o en sí tiene un fondo para cualquier contingencia, puede brindar a emprendedores y dueños más estabilidad y 
seguridad para garantizar un futuro a corto y mediano plazo. De acuerdo con una encuesta realizada por la Comisión Europea (CE) en colaboración con el Banco Central Europeo (BCE), en 2011 la mayoría de las empresas apuntaba las dificultades de acceso a un financiamiento como la segunda mayor preocupación, después de la búsqueda de clientes (Peña et al., 2017). Es importante mencionar porqué las PyMEs necesitan financiamiento. Dentro de las razones están la apertura de la empresa, la mejora de operaciones, la expansión, etcétera. De acuerdo con una encuesta publicada en 2017 por MSME Finance Gap (2019) "la demanda total de financiamiento de las PyMEs alcanza los \$2,150 millones de dólares, mientras que la oferta total de fondos de las instituciones financieras es de \$347 millones de dólares". Aquí se puede apreciar claramente una brecha entre la demanda y la oferta de los financiamientos para empresas pequeñas y medianas. Las instituciones financieras destinan estos fondos basados en pronósticos de préstamos, en los que solo se considera al $16 \%$ de los solicitantes como candidatos a recibir recursos para sus negocios y el $84 \%$ restante no los obtiene.

Se considera alarmante el número de financiamientos rechazados y no otorgados debido a que los emprendedores se ven orillados a buscar otra forma de conseguir esos recursos requeridos. Se menciona que el financiamiento a las MIPyMES es una de las principales prioridades para los gobiernos de todo el mundo en materia de políticas, y muchos de ellos han realizado iniciativas para facilitar el acceso a distintas fuentes de financiamiento (OCDE, 2018). Si en una empresa considerada micronegocio, pequeña o mediana se realiza una búsqueda en internet con el tema "apoyo del gobierno a PyMEs", se obtendrán múltiples opciones.

La literatura nos dice que, según los autores Vecino y Boggio (2006), "a pesar de la eficiencia de los programas de apoyo en términos de cobertura y atención a empresas es importante resaltar que la falta de indicadores que permitan evaluar la eficiencia de los programas dificulta el análisis". Se pretende, dentro de esta investigación, encontrar los diversos métodos por los cuales los micronegocios y las pequeñas y medianas empresas se financian, así como analizar cuáles son los financiamientos más concurridos y convenientes para impulsar este importante sector de la economía mexicana.

\subsection{Definición del problema}

Uno de los principales retos para las MIPyMES en México es el acceso a un financiamiento, como ya fue mencionado. Estas empresas han enfrentado este problema por décadas, pero cuando se han presentado crisis, la situación se agrava cada vez más para los otorgadores de estos créditos. Por lo regular, las MIPyMES acuden a un financiamiento externo mediante instrumentos que pueden ser de deuda a corto plazo o préstamos bancarios. Cuando estos proyectos son analizados por estas entidades, puede ser que la percepción que se tenga acerca de ellos sea de un gran riesgo, sobre todo en proyectos que incluyen innovación y entrada a mercados internacionales (Rojas, 2017).

Debido a las limitaciones que las MIPyMES tienen frente al financiamiento, es claro que se identifica un problema que aún no ha tenido solución y que, a pesar de todos los programas ofrecidos para impulsarlas por parte del gobierno y empresas, aún queda un gran camino que recorrer. Su importancia nace en las contribuciones que tienen estas empresas para el país y en todos los sectores que impactan.

Muchas de las restricciones en el otorgamiento de recursos a estas empresas surgen de su fragilidad en el mercado. Al respecto, Hernández y Albella (2017) mencionan que "ante los cambios de ciclos económicos se han reforzado políticas públicas que enfocan esfuerzos en la promoción e implementación de subsidios, incentivos a tasas de interés, líneas de crédito especiales que no necesariamente cumplen con el propósito de reactivar el segmento y darle sostenibilidad a largo plazo". 


\subsection{Pregunta de investigación}

Tomando como base los antecedentes presentados en Peña, Rojas y Guijarro (2017), el presente trabajo consiste en identificar las diversas formas de financiamiento para los microempresarios mexicanos. Por lo tanto, la pregunta principal del estudio es la siguiente: ¿Cuáles son las formas de obtener un financiamiento para las microempresas?

\subsection{Objetivos de la investigación}

Objetivo general:

Conocer las diversas formas de

financiamiento de las microempresas mexicanas.

\section{Objetivos específicos:}

1. Identificar los métodos de financiamiento más concurridos en México.

2. Analizar las principales razones por las cuales se solicita el financiamiento en México.

\subsection{Justificación}

La importancia del financiamiento en una empresa es relevante en diversos aspectos, por ejemplo, cuando la empresa busca crecimiento, expansión, innovación y la mayoría de las veces no cuenta con los recursos necesarios para hacerlo y su única opción es recurrir con alguna entidad a solicitarlos. Cuando se solicitan estos financiamientos, una cantidad de requerimientos serán pedidos al demandante $\mathrm{y}$, en muchas ocasiones, las MIPyMES no cuentan con esa información. Parte de los requerimientos no existentes pueden ser estados financieros actualizados, activos intangibles no registrados o carecer de un aval o garantías, entre otros.

Dada su importancia, fomentar el crecimiento de las MIPyMES tiene beneficios múltiples. El gobierno conoce bien estas necesidades, por lo que también cuenta con programas de apoyo a las empresas que requieren financiamiento. Es importante mencionar que no todas reciben estos apoyos dado que, incluso el gobierno, emplea métodos para medir el riesgo de las MIPyMES que solicitan el préstamo.

\subsection{Viabilidad}

En este trabajo de investigación, la ENAMIN (encuesta a micronegocios) del año 2012 hace posible que el análisis de la procedencia del financiamiento de las MIPyMES en México. La encuesta comprende XXII secciones, la sección XIX corresponde al financiamiento y abarca de la pregunta 80 a la 89 . Con esta data se podrá cumplir el objetivo de este estudio debido a que se podrá saber de dónde viene el financiamiento de las MIPyMES en el país y cuál de ellos es el más concurrido.

\subsection{Limitación y delimitación}

La encuesta utilizada en esta investigación es la ENAMIN del año 2012, creada por el Instituto Nacional de Estadística y Geografía (INEGI). Esta encuesta fue diseñada $\mathrm{y}$ recolectada ocho años atrás, lo que significa una limitación dado que no se cuenta con la información más actualizada. Esto puede tener una influencia en la situación económica actual debido a que desde ese año la economía debió de haber experimentado cambios y fluctuaciones. Sin embargo, es la base de datos más reciente para este estudio dado que no ha habido actualizaciones en los últimos años.

Las delimitaciones de este proyecto de investigación incluyen la especificación en términos concretos de nuestras áreas de interés, los cuales son poder conocer de dónde viene el financiamiento que utilizan las MIPyMES en México. Como se mencionó en el objetivo anterior, se pretende conocer cuáles son los métodos utilizados para acceder a recursos monetarios para diferentes propósitos, dependiendo de la empresa. El alcance de este proyecto es a nivel nacional, dado que gracias a la encuesta ENAMIN (2012) se podrá tener un análisis de esta magnitud. Este proyecto se realizará en un 
tiempo total de tres meses, el cual es suficiente para llevar a cabo un amplio estudio de los objetivos explicados anteriormente. .

\section{MÉTODO}

Este apartado está conformado por la metodología y el diseño de la investigación implementada para poder obtener los resultados acerca de las hipótesis establecidas y así poder llegar al análisis de la procedencia del financiamiento de las MIPyMES en México, así como la trata de los datos para obtener los resultados. Para cumplir con nuestro objetivo principal, se recurre a la encuesta a microempresas en México llamada ENAMIN 2012 (Encuesta Nacional de Micronegocios) implementada por el Instituto Nacional de Estadística y Geografía (INEGI). Esta encuesta está regida por el artículo 37 de la Ley del Sistema Nacional de Información Estadística y Geográfica.

\subsection{Metodología de la investigación}

La investigación por llevarse a cabo será del tipo cuantitativa y correlacional. En esta se busca conocer el grado de las variaciones de los factores con relación a otros factores para determinar de dónde proviene el financiamiento de las MIPyMES en México. Se busca también conocer la frecuencia con la que se recurre a los métodos de financiamiento, previamente mencionados en la revisión de la literatura, así como si el financiamiento fue obtenido o no, los montos de los préstamos y la mensualidad pagada por ellos.

La investigación es cuantitativa, lo cual nos permitirá conocer la realidad de una manera más imparcial debido a que se recogen y analizan los datos a través de los conceptos y variables estudiados en la encuesta ENAMIN 2012. Esta base de datos brindada por el INEGI encaja de manera perfecta para los propósitos de esta investigación.

La ENAMIN es un estudio a microempresas y pequeños negocios que tienen desde seis hasta 16 empleados, incluyendo al propietario del establecimiento. Teniendo esta base de datos será posible hacer un análisis de la situación económica de las MIPyMES en México en cuanto al financiamiento. La muestra de la encuesta es de 30,063 empresas de diferentes tipos de microempresas en México. Como se mencionó, estas empresas son de diversas industrias, las cuales incluyen minería, construcción, comercio, servicios, transporte, manufactura, entre otros. Es importante mencionar que dadas las limitaciones del mercado, los emprendedores en su mayoría optan por tener negocios informales. Cuando una empresa es informal significa que no paga impuestos o tarifas de seguros al gobierno. En México, solo el $26.69 \%$ de las empresas son formales, el $73.31 \%$ restante están operan en la informalidad (Haro, 2015). Cabe mencionar que la informalidad es otro factor que dificulta el acceso a financiamientos, dado que las empresas no cuentan con información para medir su capacidad crediticia o la del emprendedor.

Se utilizará el sistema SPSS para obtener los resultados buscados y poder interpretar la situación actual de México en cuanto a financiamientos. La base de datos fue obtenida y transportada al sistema para así poder establecer los comandos necesarios para mostrar gráficamente lo recabado en la encuesta.

\subsection{Diseño de la investigación}

En este estudio se elaboró un diseño no experimental, ya que se limita a la observación de la conducta de las variables que se desean explicar sin sufrir manipulación, es decir, tal y como sucedieron en su contexto natural. La investigación tiene una cohorte transversal o transeccional pues analiza la información estadística para describir y explicar las relaciones existentes entre las variables en un punto o momento de tiempo a estudiar (Hernández et al., 2016).

\subsection{Hipótesis}

A continuación, se mostrará la hipótesis en la cual está basada esta investigación: 
$H_{1}$ El financiamiento de las MIPyMES en México proviene de fuentes externas a instituciones establecidas como la banca y SOFOM.

\subsection{Fuentes de información}

La presente investigación analiza la información nacional recolectada por el INEGI por medio de la Encuesta Nacional de Micronegocios (ENAMIN) para el año 2012, en los sectores económicos de manufactura, comercio, construcción y servicios de todo el país.

La encuesta diseñada por el INEGI es de tipo estructurada y se diseñó para obtener información de las microempresas en las que laboren de una a seis personas incluyendo al dueño y hasta 16 si se trata de empresas en el sector manufacturero. La encuesta se aplica utilizando la técnica de encuesta estructurada, administrada y personal a nivel nacional.

La ENAMIN se ha aplicado en ocho ocasiones en los siguientes años: 1992, 1994, 1996, 1998, 2002, 2008, 2010 y 2012. Los cuestionarios para los ocho años son ligeramente diferentes en los distintos años de su aplicación. También varían el número de empresas que el INEGI encuestó en cada levantamiento de información. Estos cuestionarios tienen variaciones que van de las 71 a las 100 preguntas según el año de su aplicación. Como se ha mencionado, la presente investigación estudia a nivel nacional los resultados correspondientes al año 2012. En esta tesina sólo se trabaja con las secciones y las preguntas de la ENAMIN que resultaron del eje temático de financiamiento; en el Anexo 1 se muestra dicha sección.

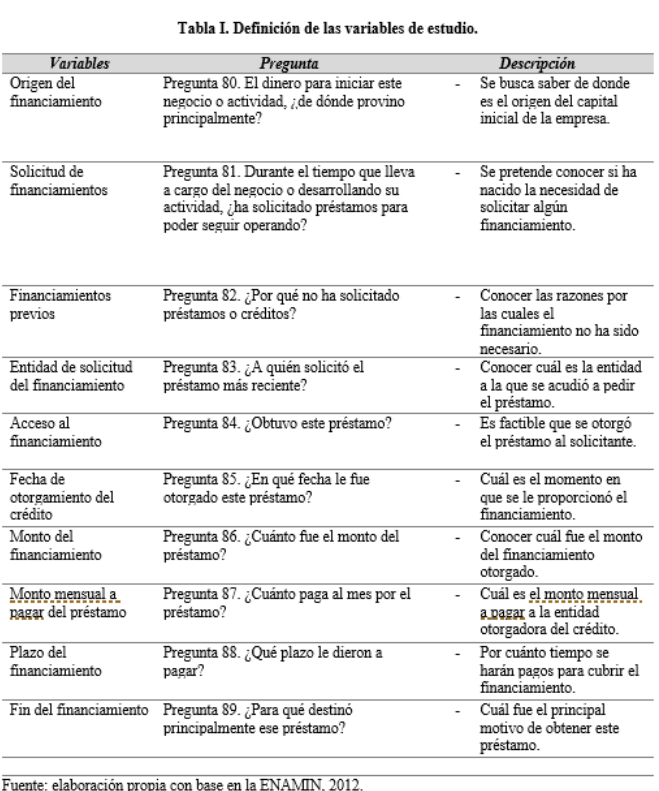

\subsection{Variables}

En la siguiente sección se explicarán cada una de las variables en este estudio, las cuales fueron ilustradas en la Tabla 1. La primera de ellas es el origen del financiamiento, que se muestra en la pregunta 80 del cuestionario y consiste en conocer de dónde vino el capital inicial para comenzar con el negocio. Este capital puede provenir de la banca comercial, ahorros, liquidaciones o, en algunos casos, el negocio es heredado. La segunda variable es la solicitud del financiamiento, la cual muestra si las MIPyMES han tenido la necesidad de pedir un financiamiento con alguna entidad externa. Los financiamientos previos es la tercera variable mostrada y hace referencia a tener en consideración los motivos por los cuales no se han solicitado préstamos. Para esta variable puede haber una variedad de respuestas tales como: no se tiene la necesidad, muchos intereses, etcétera. La cuarta variable es la entidad de solicitud del financiamiento que consiste en saber cuál es la entidad o persona a la que la MIPyME acudió a pedir financiamiento. Continuamos con acceso al financiamiento como quinta variable, la cual refiere a qué tan factible fue que se le otorgara el préstamo a la microempresa. Fecha de otorgamiento del crédito se posiciona como la 
sexta variable de este proyecto, y extrae información del mes y el año en que el préstamo fue otorgado. La séptima variable es el monto del financiamiento y provee un rango de los montos que las empresas han adquirido para reinversión o como capital inicial. Seguimos con monto mensual a pagar del préstamo, para continuar con el plazo del financiamiento, las cuales son variables que comprenden los términos del financiamiento, así como su fin u objetivo.

\section{RESULTADOS}

Para continuar con este proyecto, analizaremos los resultados obtenidos de los hallazgos descubiertos en la encuesta. Estos hallazgos están concentrados en la forma de financiamiento de las MIPyMES en México. En este capítulo se muestra de manera gráfica y escrita la situación en la que se encuentra el país en este tema en particular. Asimismo, se podrá argumentar si la hipótesis de este proyecto se cumple bajo el estudio realizado.

\subsection{Presentación de los resultados obtenidos}

Esta sección se basará en los resultados obtenidos con el estudio, el cual tiene por objetivo correr un análisis acerca de la procedencia del financiamiento de las MIPyMES en México. Con esto se podrá tener una imagen de la situación actual del país en materia de apoyo a las micro y pequeñas empresas que son el principal motor de la economía. Es probable que estos resultados sean de gran impacto para el sistema economico de México, dado que pueden despertar gran interés en algunas partes involucradas.

\subsection{Estadísticos descriptivos de la base de datos}

Para comenzar con los resultados, haremos una descripción de las personas encuestadas que se tomaron el tiempo de contestar la ENAMIN con más de 100 preguntas a responder. Es importante mencionar que tener conocimiento de los datos generales de estas personas, en su mayoría dueños de alguna micro o pequeña empresa o emprendedores, también podrá darnos una idea respecto de si los negocios fueron emprendidos o abiertos recientemente. A su vez, este argumento refiere a si los negocios en este país se encuentran tanto en la formalidad como en la informalidad. Se comenta que, a pesar de las recientes recuperaciones con el crecimiento económico, la apertura de múltiples centros comerciales está destruyendo puestos de trabajo que en su momento son formales, y aumentando, como consecuencia, el empleo informal (Martínez, 2005).

En la siguiente gráfica se puede observar una ligera diferencia entre el género de las personas entrevistadas que no necesariamente son los dueños del negocio, sino algunos de los trabajadores que en ese momento realizaron la encuesta solicitada. Se puede decir que en el pasado, en su mayoría, los emprendedores eran hombres dedicados a sacar adelante a sus familias $o$ en búsqueda de crecimiento profesional o económico. En el siguiente gráfico se muestra un hallazgo importante. Hay una diferencia entre el género femenino y el masculino de 191 personas que contestaron esta pregunta. Es solamente una pequeña diferencia entre ambos géneros, lo cual indica un avance en la sociedad en general. Nos comenta García (2019) que "en México, las mujeres tienen mas retos para poder crear mantener y crecer una empresa". Regresando al tema de la informalidad, se comenta que la mayoría de las mujeres emprendedoras trabaja bajo este concepto, dado que los negocios creados por ellas surgen principalmente por necesidad y no de manera voluntaria (García, 2019). Es un importante indicador que hoy en día para comenzar una MIPyME, todas las personas tienen las mismas oportunidades de sacar adelante un emprendimiento. 


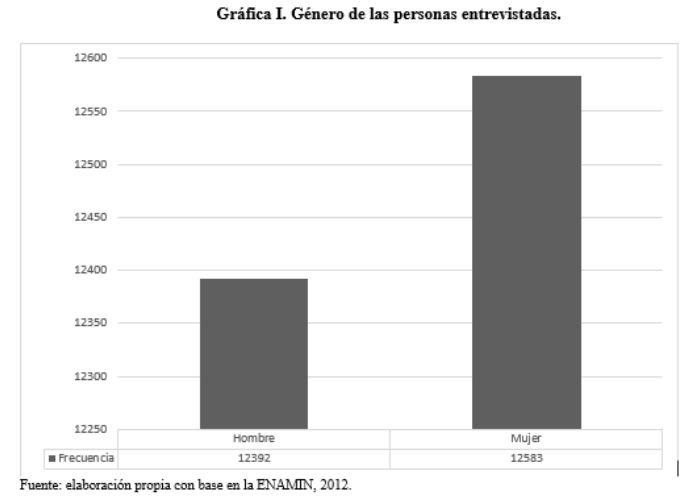

Con referencia a la edad de las personas encuestadas, se puede observar que la mayoría recae sobre los 46 años en adelante, en lo que corresponde a quienes contestaron esta pregunta. Este es un indicador que hace referencia a que las MIPyMES, en su mayoría, son negocios que tienen muchos años en operación. Es importante mencionar que también se puede ver una tendencia en donde las nuevas generaciones están tomando el emprendimiento e impulsándolo desde muy jóvenes. No está establecida cierta edad para comenzar un negocio, dado que esto puede ocurrir desde edades muy tempranas; se observa que 1,542 de las personas encuestadas se encuentran entre los 12 y 25 años de edad.

Hay muchos autores que indican, por ejemplo López (2013), que la edad perfecta para comenzar a emprender es a partir de los 35 años de edad. Se argumenta que las personas de esta edad tienen los conocimientos, las experiencias, la paciencia y la madurez necesaria para lograr el éxito con los emprendimientos. Si tomamos como referencia a algunos emprendedores famosos, tales como el creador de la plataforma de Facebook, Mark Zuckerberg, o Steve Jobs, el creador de la marca Apple, son excepciones a lo anteriormente mencionado. La edad de entre los 20 y 30 años puede ser considerada como apropiada dado que las personas emprendedoras pueden tener las ganas, la energía y el entusiasmo para sacar el negocio adelante, pero sin la experiencia que probablemente no tenga que ser necesaria. En conclusión, no hay una edad establecida que las personas tengan que alcanzar para lanzarse a cumplir un sueño de emprendimiento.

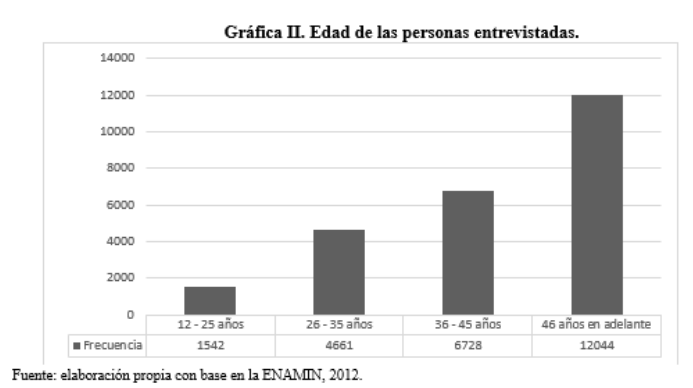

Se puede observar en el gráfico siguiente que la mayoría de las personas encuestadas se encuentra en un estado civil casado, seguido por quienes viven en unión libre con sus parejas. Es importante mencionar que cuando una persona tiene la responsabilidad de una familia, es mayor el ímpetu por salir adelante y entrar en el mundo del emprendimiento para generar los ingresos suficientes para las necesidades familiares dado que hay personas que dependen del emprendedor.

También, es evidente que las personas con estado civil de soltero(a) forman gran parte de estos emprendedores. Se puede mencionar que dado que la mayoría no tiene una responsabilidad fija, como familia, deudas, etcétera, puede invertir sus recursos en el impulso o creación de nuevos proyectos aceptando el riesgo que puedan llegar a representar. Actualmente, muchas carreras universitarias cuentan con materias que tienen enfoque hacia el emprendimiento o el emprendimiento social. Nos menciona el autor Canales (2007) que en la actualidad "se ha renovado el interés por el estudio del emprendimiento, enmarcado como vía para subsanar las ineficiencias presentadas en el mercado de trabajo, delimitadas por la gran demanda de fuentes de trabajo". Es de suma importancia dada la situación económica, que las personas tengan noción de lo que es emprendimiento y todo lo que puede llegar a abarcar esta disciplina. Por su parte, González (2017) nos menciona que "se debe responder a la esencia que tiene esta disciplina para poder reconocer oportunidades de negocio y como es que estas son identificables". 


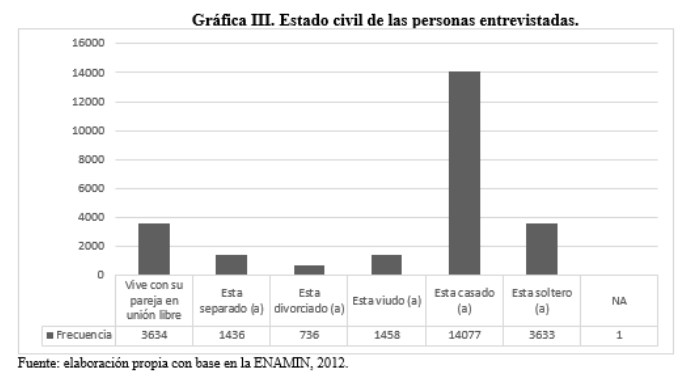

Dado que la ENAMIN (2012) está enfocada en las micro y pequeñas empresas, en la siguiente gráfica se puede observar que la mayoría de estos negocios tiene menos de 10 empleados, incluyendo al dueño. Se puede ver que la mayoría de ellas, con 5,429 respuestas, tiene dos personas laborando, y en esto se considera al empleado y al dueño como trabajadores. Por otro lado, nos comenta la autora Moreno (2009) que para el año 2012 se hizo una reclasificación en la que el tamaño que tuviera la empresa dependería directamente del número de empleados y del monto de ventas anuales por $90 \%$. Se puede ofrecer el siguiente rango de estratificaciones.

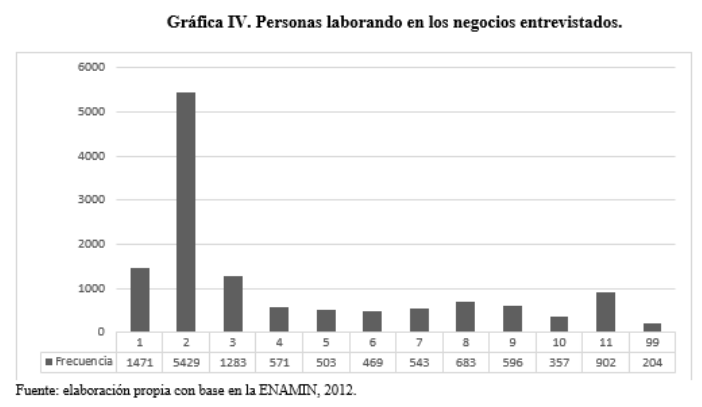

Una de las características relevantes para estos negocios MIPyMES es que en su mayoría son emprendidos por una persona y solamente 1,095 se componen de varios dueños. Es importante mencionar que cuando una persona emprende solo o con otras personas, cada una de estas opciones tiene ventajas y desventajas. Para el caso de las MIPyMES, cuando se emprenden por una sola persona, la principal desventaja es que se asume todo el riesgo de la pérdida del capital que se está invirtiendo en la creación del proyecto. En cambio, si varios socios proveen el capital, todos tomarán la responsabilidad en caso de que no salga bien el proyecto, dependiendo de la cantidad que hayan aportado al mismo.

Cuando se tiene negocio de un solo dueño, esa persona tiene el dominio total del mismo y es la única responsable; por consiguiente, el beneficio económico será solamente para una persona y no estará dividido entre varios socios. Cuando la empresa tiene más de un socio, cuenta con la ventaja de que el capital inicial invertido pueda ser mucho mayor a lo que solo un socio pueda aportar. Las ganancias serán repartidas por partes acordadas entre los socios, en caso de que sea más de uno. Dependiendo de la decisión que se tome, estas ventajas y desventajas deben ser evaluadas para tomar la que mejor beneficie a todas las partes interesadas.

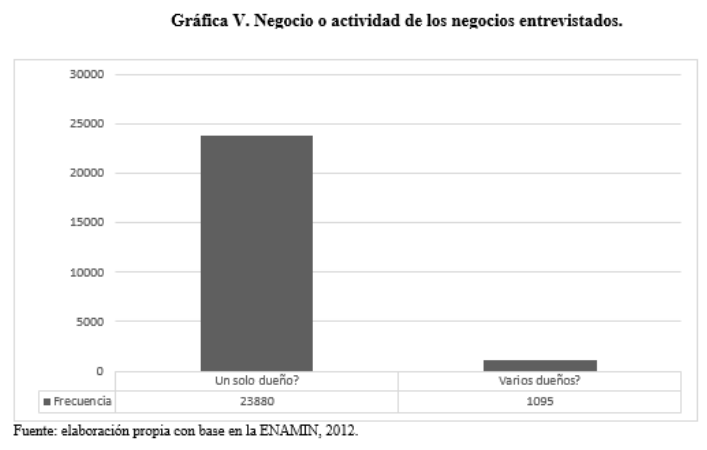

Es importante mencionar, como se ven el gráfico debajo de este texto, que la mayoría de estos negocios son familiares. Dentro de esta pregunta está la posibilidad de que el negocio haya sido heredado por generaciones dentro de la misma familia. Hay MIPyMES que han logrado sobrevivir a lo largo de los años por el éxito que han tenido o la popularidad adquirida en las zonas geográficas en las que se localizan. Esto puede ser considerado como éxito, dado que la esperanza de vida que tienen las empresas MIPyMES en México es de aproximadamente 7.8 años a partir de su nacimiento, y esto varía dependiendo del sector. Se comenta que, de acuerdo con el Censo realizado por el INEGI en el año 2019, aproximadamente el 39\% de los negocios ya no estaba cuando los encuestadores regresaron a visitarlos (Rodríguez y Saldívar 2020). 


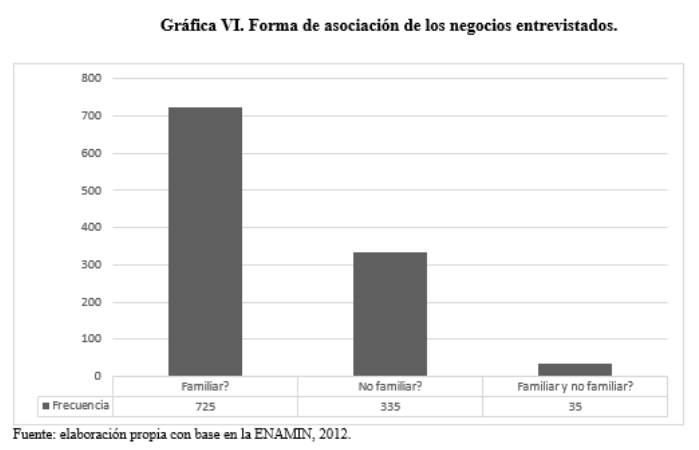

\subsection{Análisis de las frecuencias de las variables}

A continuación, se hará un análisis de las preguntas que están enfocadas en el financiamiento de las MIPyMES encuestadas en México. Los resultados obtenidos nos brindarán un panorama que podrá posicionar la situación económica en la que se encuentra el país en el ámbito del financiamiento. Es importante recordar que la falta de financiamiento tiene repercusiones en las empresas que pueden ir desde una quiebra financiera que puede generar, en el peor de los casos, deudas a largo plazo y problemas legales acompañados del cierre del negocio.

Comenzando con el análisis, la Gráfica 7 ilustrará de dónde provino el dinero inicial para comenzar el negocio MIPyME. En la gráfica se puede observar que, en su mayoría, estos negocios fueron impulsados por el ahorro personal de los mismos dueños. No se puede tener un dato de cuánto tiempo le tomó al emprendedor recaudar la cantidad requerida para poder arrancar operaciones en la MIPyME, tampoco si fue la cantidad necesaria para hacerlo. También, es importante tomar en cuenta el tamaño de la inversión si un proyecto es grande, considerando el factor de riesgo de pérdida de la inversión en caso de que los proyectos no tengan el éxito deseado.

Por consiguiente, se puede observar que estos ingresos también vienen de préstamos de amigos o familiares, lo cual es una opción muy común para evitar así el pago de intereses generados. Estos préstamos, en su mayoría, no requieren ninguna garantía o aval y tienen la ventaja de que los plazos otorgados y los pagos pueden ser cómodos para los emprendedores. Con porcentajes bajos dentro de la encuesta, se encuentran los préstamos que vienen de la banca, de cajas populares, crédito de proveedores, etcétera. De aquí nace la importancia de este estudio, dado que como los porcentajes de estos préstamos son bajos, es de gran interés analizar qué hacen los emprendedores para conseguir los recursos necesarios para impulsar los proyectos. Se puede observar que estos recursos también son producto de liquidaciones de empleos anteriores o de venta e hipoteca de bienes. Algunos otros heredaron el negocio, como se comentó en párrafos anteriores.

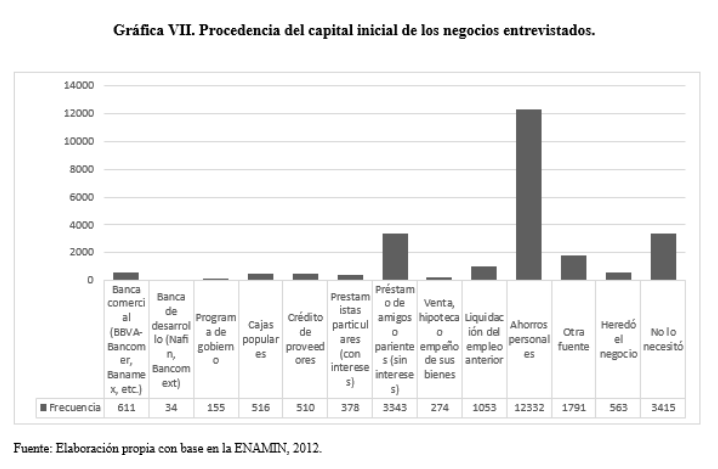

En algún momento de la vida de los negocios surge la necesidad de innovación o crecimiento. Esto se debe a que, a medida que pasa el tiempo, las cosas van cambiando y la forma en que se hacen los negocios también. Es importante que los dueños de estos negocios estén al pendiente de las innovaciones y nuevas tecnologías que van naciendo a través del tiempo. Es por eso que para llevar a cabo cualquiera de estas opciones, se necesitan recursos monetarios.

Se puede poner de ejemplo a los negocios dedicados al sector de alimentos en la pandemia ocasionada por el COVID-19 en el año 2020. Dado que por instrucciones de las autoridades la población estuvo obligada a quedarse en casa, fue necesario que los restaurantes pudieran ofrecer a los consumidores servicio a domicilio y la solución que encontraron fue afiliarse a las plataformas como UberEats, DiDi Food, Rappi, etcétera. Algunas de estas plataformas establecen una tarifa inicial, no tan altas, lo cual es parte de esta innovación que los 
negocios tienen que alcanzar.

En la siguiente gráfica se puede observar que, dentro de los negocios encuestados, la mayoría no ha solicitado préstamos para seguir operando. Dentro de las razones por las cuales las MIPyMES no han solicitado estos préstamos es porque no han tenido la necesidad de hacerlo. Esto puede ser un indicador de que el negocio ha generado el suficiente capital para sostenerse y soportar los costos de las mejoras. Algunos negocios siguen con el mismo sistema con el que empezaron a operar y este no ha necesitado ningún tipo de innovación, lo cual es un caso. Pero para muchas otras MIPyMES a veces es necesario el cambio para alcanzar a la competencia y no quedarse detrás.

La segunda de las razones es que a estas personas no les gustan endeudarse o pedir prestado dado que las comisiones o intereses generados son muy altos. Esto mismo es otra de las razones de porqué no se solicitan estos préstamos. Otras de las razones incluyen respuestas como no saber cómo pedirlo o básicamente estos dueños no saben cómo solicitarlo a una entidad financiera. Todas estas dudas o preguntas pueden jugar un papel importante en el desarrollo del negocio en el siguiente nivel, para alcanzar a la competencia o simplemente para su sobrevivencia.

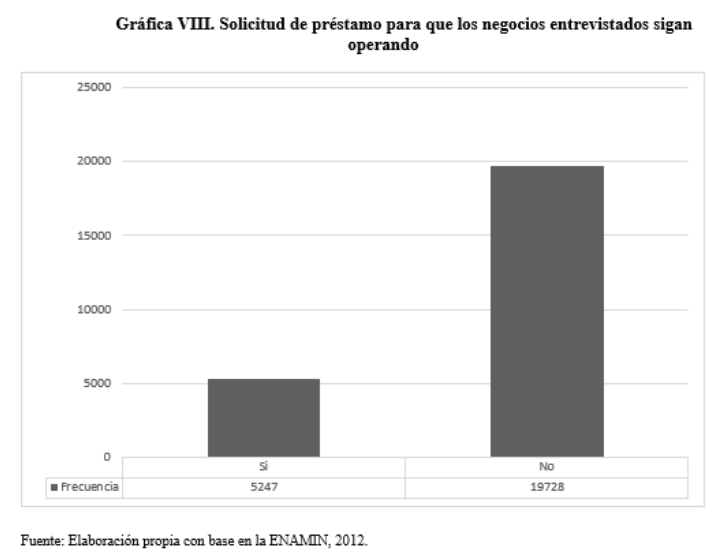

Para complementar lo argumentado anteriormente, se puede observar en el siguiente gráfico que los dueños de las MYPyMES no saben pedir financiamientos, piensan que no se los otorgarán, no les convienen, los intereses son muy altos, los trámites son largos y cansados, y no podrían pagarlos. Es alarmante que en el país no se tenga una cultura de crecimiento que impulse a los emprendedores a tomar el reto para crear nuevos negocios que significan para el país nuevas fuentes de empleo e ingresos. Una cultura de emprendimiento, según Hidalgo (2015), ha nacido de las crisis económicas que ha vivido la sociedad con la falta de empleo que, a la vez, demanda profesionales más eficientes, competitivos y multidisciplinarios. Es por eso que una inversión en algún proyecto puede representar un reto desafiante para muchos emprendedores.

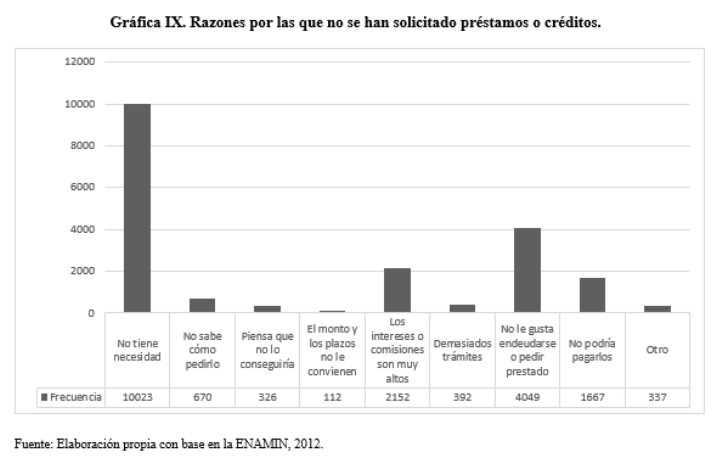

En caso de que estos emprendedores sí hayan solicitado el préstamo, el siguiente gráfico nos muestra a quién se solicitó el préstamo más reciente. Entre las opciones de respuesta están los bancos, gobierno, cajas populares, prestamistas particulares, amigos y familiares. Se observa que la gran mayoría de estos negocios acudió a una banca comercial a solicitar el préstamo, seguido por cajas populares. Es importante mencionar que el proceso que se toman estas instituciones es, en su mayoría, muy burocrático y rudimentario.

Estos procesos solicitan del demandante cierta información con la que no cuentan en muchas ocasiones, por ejemplo, estados financieros. Para las MIPyMES es muy complicado tener esta información porque, debido a su tamaño, posiblemente no cuentan con la contabilidad. Aunque es probable que las empresas que ya tienen más años y han crecido cuenten con sistemas de contabilidad que les permitan tener esta información. Por otro lado, es importante recalcar que los dueños pueden 
recurrir como mejor opción a obtener el financiamiento como persona física que a nombre de la empresa.

Enseguida, en la gráfica analizada, se encuentran los préstamos entre amigos o parientes como los más concurridos. Se mencionaba anteriormente que este tipo de préstamo es, para muchos emprendedores, más cómodo, dado que los plazos y montos de los pagos son flexibles debido a la confianza que existe entre el prestamista y el emprendedor. Después, tenemos a los prestamistas particulares que sí cobran intereses y pueden solicitar plazos un poco más limitados para varios emprendedores. Es importante comentar que un bajo porcentaje de la muestra acudió a los programas de gobierno que ofrecen apoyo a las MIPyMES de México. Para el año 2020, se destinó en el país un presupuesto de apoyo a estas micro, pequeñas y medianas empresas. Este presupuesto aumentó un 20\% comparado con el año anterior (2019). Para este año 2021, en el documento entregado por la Secretaría de Hacienda y Crédito Público (SHCP) a la Cámara de Diputados se propusieron 3,503 millones de pesos para las MIPyMES, mientras que en 2020 se propusieron 2,929 millones de pesos mexicanos (Meza, 2020).

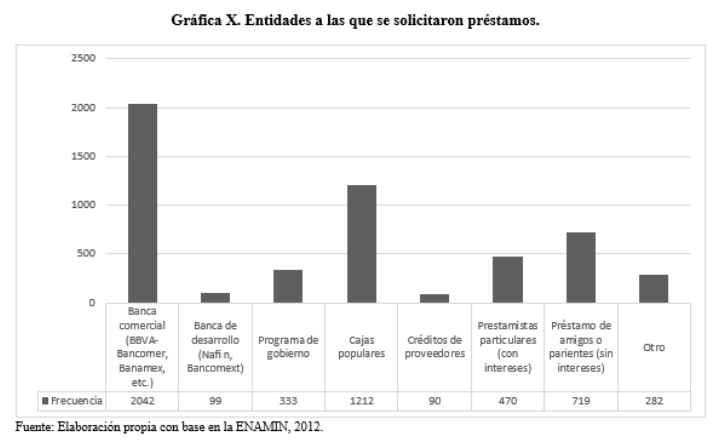

Se puede observar que de los préstamos solicitados, la mayoría fueron otorgados. Solamente 244 de las personas que solicitaron un préstamo contestaron que no se les otorgó. Un punto importante de mencionar es que sí se está apoyando a las MIPyMES que solicitan préstamos para crecimiento y expansión. Teniendo en cuenta las más de 30 mil respuestas en esta encuesta, cinco mil créditos otorgados representan la sexta parte de la muestra en realidad. Habría un escenario diferente si todas estas empresas pudieran tener acceso a un crédito para mejorar o en algunos casos subsistir.

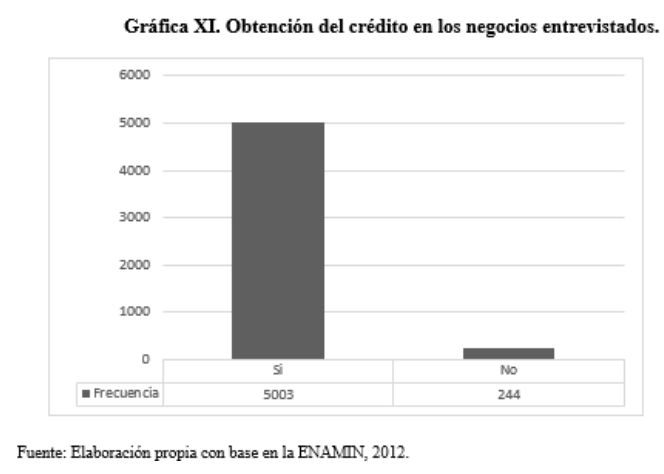

Dentro de los rubros del préstamo o financiamiento, se puede observar que la mayoría de los negocios solicitó un préstamo de menos de 10,000 pesos mexicanos, una cantidad baja considerando la inversión que la MIPyME esté tomando en cuenta. Por consiguiente, se puede ver que el pago mensual de estos préstamos, en su mayoría, es menor de 1,000 pesos mexicanos. Solamente 258 negocios tienen mensualidades que abarcan de 5,001 a 10,000 pesos y otros 131 negocios pagan más de 10,001 pesos mexicanos.

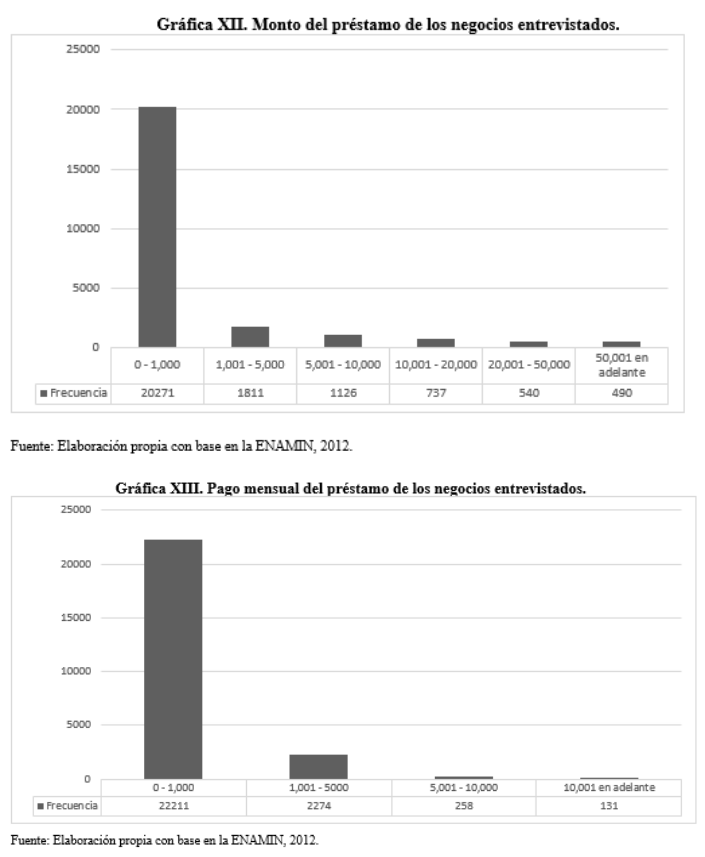

En el siguiente gráfico se puede observar en 
detalle cuál es el destino de los recursos que se piden a entidades bancarias para los negocios. Principalmente, se puede observar que estos recursos son utilizados para adquirir o comprar nueva mercancía, seguido por la compra de maquinaria, equipo o herramientas necesarias. Entre otras opciones por las cuales se utilizan estos recursos se encuentran para comprar local, vehículos, hacer alguna ampliación del negocio, etcétera. También, dentro de los resultados se puede ver que estos préstamos han sido destinados para pagar deudas anteriores.

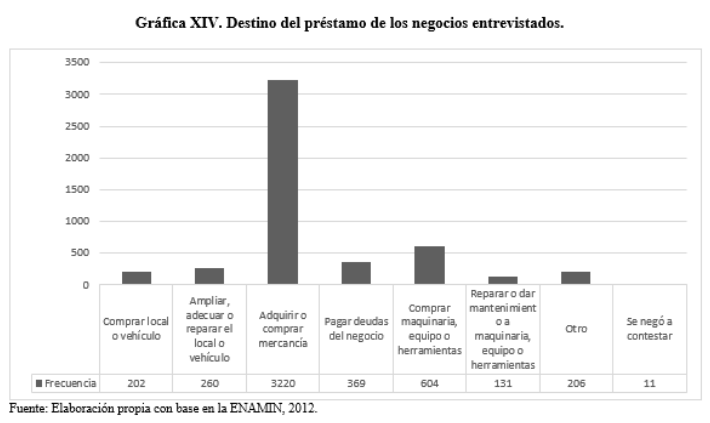

Cualquiera que haya sido el destino de estos fondos, el financiamiento representa para las MIPyMES una nueva oportunidad y el hecho de que este pueda ser otorgado tiene repercusiones muy importantes en la economía mexicana y en la mejora de muchos de los indicadores acerca del estado en el que se encuentra el país actualmente.

\subsection{Discusión de resultados}

Este estudio tiene una aplicación no experimental para las MIPyMES en México con base en la encuesta ENAMIN (2012) conducida por el INEGI. En el estudio, se puede observar una muestra que está dentro del sector micronegocios, pequeñas $y$ medianas empresas, a las cuales se les aplicó un cuestionario de más de 100 preguntas para obtener información de varios ámbitos que las involucran. Dentro del análisis de los resultados, se puede observar claramente que estas empresas son negocios con menos de 10 empleados que pueden tener más de un dueño a la vez, ya sea de género masculino o femenino con un rango de edad variado.

En lo que respecta al financiamiento de las MIPyMES, se puede observar claramente que para México existen varias opciones de mejora. En esta economía en desarrollo tiene que darse más valor a estas empresas que representan el motor del país. Se ha mencionado, en repetidas ocasiones, lo que ellas representan para las tasas de empleo y, más importante aún, para el PIB (Producto Interno Bruto) anual. Es tanta la importancia de las MIPyMES que si desaparecieran, la economía tendría un impacto muy significativo.

Es importante mencionar que también muchas de las MIPyMES mueren en sus primeros años de vida al no poder ir al mismo nivel que lo demanda la economía, y con esto nos referimos a que probablemente no cuentan con las herramientas ni el capital para hacerlo. La tasa de mortalidad en las MIPyMES debería ser un asunto vital para nuestro gobierno, el cual está ofreciendo más de tres mil millones para apoyar a estas empresas, que son destinados a negocios que tienen el conocimiento de estos fondos y cumplen con todos los requisitos para solicitar el préstamo. Crear una cultura en la que la MIPyME pueda tener conocimientos de las opciones y oportunidades que tienen estos negocios para innovar sería un gran cambio en la economía y generaría un impacto económico y social. Cuando hablamos de impacto económico, este cae directamente en el PIB, y cuando se habla de un impacto social es porque la sociedad estará adquiriendo la cultura del emprendimiento y poniéndola en acción.

\section{CONCLUSIONES}

\subsection{Conclusiones}

Este estudio tiene una aplicación no experimental para las MIPyMES en México con base en la encuesta ENAMIN (2012) conducida por el INEGI. Esta encuesta incluye casi 30,000 muestras de entrevistas que se les hicieron a dueños de estas empresas en el país. Esta muestra fue tratada en SPSS para así 
poder tener frecuencias, porcentajes, etcétera, y poder correr un análisis de los resultados obtenidos. Es importante mencionar que la ENAMIN (2012) fue utilizada para obtener información de la procedencia del financiamiento de las MIPyMES y otras variantes que nos pueden brindar información valiosa del mismo.

Con base en el análisis de resultados, se mostró que, en general, el financiamiento es un tema que tiene muchas oportunidades de mejora en cuanto a los micronegocios y pequeñas empresas. Este tema también tiene mucho impacto en cuanto a la salud financiera y el crecimiento. De acuerdo con los autores Verbano y Venturini (2013) "la adopción de la administración del riesgo de crédito ayuda a las empresas a reducir la incertidumbre en su desempeño, aminorando su probabilidad de bancarrota en un periodo de tiempo determinado". Este estudio tiene como primer objetivo la contribución a la investigación de riesgo de crédito en las MIPyMES, dada la falta de financiamiento que existe para este segmento de negocios. Es importante mencionar que estas empresas tienen una alta probabilidad de quebrar o ir a la bancarrota en el próximo año.

Con este estudio se puede concluir que la hipótesis se cumplió, dado que el financiamiento de la mayoría de las MIPyMES proviene de fuentes externas a entidades bancarias, SOFOMES, etcétera.

Debido a que estas empresas representan el más grande segmento de negocios y es el más grande empleador a nivel mundial (UNAM, 2015), es fundamental que se empiece a mejorar y reforzar su administración con el tema del financiamiento. Este análisis también tiene el propósito de contribuir con una alerta roja respecto a que estas empresas necesitan apoyo, no solo para México, también en la economía mundial.

Estas empresas MIPyMES son el motor de la economía, por consiguiente, se necesita que se comience a tener mejores mediciones de riesgo de crédito para las mismas que no sean tan robustas y subjetivas. Esto podrá proveerles de un mejor conocimiento acerca de su salud financiera. Tener este conocimiento puede beneficiarlas en el momento de pedir un préstamo a una entidad bancaria o cualquier institución financiera. Esto también puede beneficiar su sobrevivencia en el mercado, debido a que muchas de estas empresas tienen una esperanza de vida de menos de un año. Es importante mencionar que cuando los préstamos que sí fueron autorizados son otorgados, no se tiene el dato si fueron tramitados a nombre de la empresa o del dueño del negocio que sería considerado un préstamo a una persona física. Sería importante conocer cuántos de los préstamos otorgados a MIPyMES fueron a nombre de personas morales.

Esta investigación tiene un impacto social en la economía mexicana dado el conocimiento de las fuentes de financiamiento para los micronegocios y pequeñas empresas, que son la mayoría en el país. Los ingresos de estas empresas representan el soporte de muchas familias mexicanas, debido a que son la mayor fuente de empleo en el país. El acceso a financiamiento representa para estos negocios la oportunidad de crecimiento y la mejora en la calidad de vida para muchas familias.

Para concluir, es importante que estudios como este promuevan más investigación en este campo de estudio para un incremento de las herramientas necesarias para el manejo de medición de crédito en las empresas privadas.

\subsection{Recomendaciones}

Dados los hallazgos presentados en este estudio, claramente se pudo observar que existe una gran área de oportunidad en el tema del financiamiento a MIPyMES; se tienen que tomar ciertas medidas y recomendaciones para que estos motores de la economía mexicana sigan trabajando y subsistiendo en el periodo más largo de vida posible. Mantener estos motores de economía en operación es un beneficio a nivel nacional que el país no se puede dar el lujo de perder.

Se recomienda a los emprendedores de MIPyMES mexicanas la concientización del tema del financiamiento debido a las repercusiones positivas que puede tener en los 
negocios. Un financiamiento puede impulsar el crecimiento de la empresa o ayudarla a subsistir por un periodo determinado de tiempo. Un amplio conocimiento de las posibilidades que se tienen para financiamiento puede ayudar a las MIPyMES a elegir mejor a la hora de acudir por un préstamo, conociendo cuáles serán los requerimientos que se pedirán o los requisitos para el trámite. También, les apoyará para diferenciar opciones de financiamiento, considerando ventajas y desventajas para tomar una mejor decisión.

Sería una buena opción brindar campañas gratuitas a las MIPyMES en las que se les muestre la importancia que tienen sobre la economía y diversas maneras en las que pueden impulsar sus negocios hacia la prosperidad. Es importante que en estas campañas se incluyan los programas que ofrece el gobierno para incentivar el emprendedurismo en las MIPyMES. Estos programas ya existen y cada año se destina un presupuesto para el apoyo de estos negocios en particular.

Dentro de estos programas, parte del presupuesto gubernamental debería estar dirigido al emprendedurismo de mujeres dado que, como se mostró en el estudio, una gran parte de los emprendedores es del género femenino que no cuenta con gran apoyo económico, en su mayoría, como el género masculino.

El apoyo a las MIPyMES aseguraría un poco la estabilidad de la economía del país y su impulso tendría repercusiones positivas sobre indicadores económicos que contribuyen a la reputación que se tiene comparada con otros países, lo que puede hacer que se atraiga inversión y apoyo a la economía nacional. 


\section{REFERENCIAS}

Alvarado, E., Ortiz, J., y Morales, D. (2018). Factores que influyen en el emprendimiento de un micronegocio familiar en Mexico. Revista de Economía del Rosario, 21(1), 153-180. https://revistas.urosario.edu.co/index.php/economia/article/view/6816/6109

Canales, R. A., Román, Y. G., y Ovando, W. (2007). Emprendimiento de la población joven en México. Una perspectiva crítica. Entreciencias: Diálogos en la Sociedad del Conocimiento, 5(12),1-17. $\quad$ http://ri.uaemex.mx/bitstream/handle/20.500.11799/108908/secme23384 5.pdf? sequence $=5$ \&isAllowed $=\mathrm{y}$

García, K. (17 de febrero de 2019). Las brechas de género en el emprendimiento. El Economista. Recuperado el 10 de diciembre de 2020, de https://www.eleconomista.com.mx/economia/Lasbrechas-de-genero-en-el-emprendimiento-20190217-0001.html

González, M. (2017). El estudio del emprendimiento en México. Recuperado el 16 de diciembre de 2020 ,

http://www.acacia.org.mx/busqueda/pdf/04_PF667_Emprendimiento_en_M_xico.pdf

Hernández, G., y Albella, S. (2017). La financiación de las micro, pequeñas y medianas empresas a través de los mercados de capitales en Iberoamérica. Fundación Instituto Iberoamericano de Mercados de Valores. Recuperado el 20 de octubre de 2020, de https://www.iimv.org/iimvwp-1-0/resources/uploads/2017/03/estudiocompleto.pdf

Herrera, D. (2020). Instrumentos de financiamiento para las micro, pequeñas, y medianas empresas en América Latina y el Caribe durante el Covid-19 (Documento para discusión No. IDB-DP771). Banco Interamericano de Desarrollo. Recuperado el 13 de octubre de 2020, de https://publications.iadb.org/publications/spanish/document/Instrumentos-de-financiamientopara-las-micro-pequenas-y-medianas-empresas-en-America-Latina-y-el-Caribe-durante-elCovid-19.pdf

Instituto Nacional de Estadística y Geografía. (2009). Micro, pequeña, mediana y gran empresa. $\begin{array}{lllllll}\text { Recuperado el } & 26 & \text { de } & \text { mayo }\end{array}$ https://www.inegi.org.mx/contenidos/programas/ce/2009/doc/minimonografias/m_pymes.pdf

Instituto Nacional de Estadística y Geografía. (2014). Esperanza de vida de los negocios a nivel nacional. Recuperado el 22 de octubre de 2020, de https://www.inegi.org.mx/temas/evnm/doc/evn_ent_fed.pdf

Jurado, G. (2019). Porcentaje de pymes en México. Intuit. Quickbooks. Recuperado el 13 de octubre de 2020, de https://quickbooks.intuit.com/mx/recursos/controla-tu-negocio/cual-es-elporcentaje-de-pymes-en-mexico/

León, E., y Saavedra, M. (2018). Fuentes de financiamiento para las mipyme en México. Excelencia Administrativa, (44), 113-142. https://www.uv.mx/iiesca/files/2018/11/16CA201801.pdf

López, J. (2013). ¿Cuál es la edad perfecta para emprender? Forbes México. Recuperado el 10 de diciembre de 2020, de https://www.forbes.com.mx/cual-es-la-edad-perfecta-para-emprender/

López, V. (2018). La competitividad de las Pymes en México: Retos y oportunidades ante un mundo globalizado. Horizontes de la Contaduría en Ciencias Sociales, 5(9), 79-91. https://www.uv.mx/iic/files/2018/12/Num09-Art07-105.pdf

Luna, F., Salgado, A., Cuevas, B., y Barrios, G. (2016). Ciclo de vida organizacional en las Mipymes mexicanas, Ramo maderero. Revista de Negocios y PyMES, 2(4), 1-9. https://www.ecorfan.org/spain/researchjournals/Negocios_y_PyMES/vol2num4/Revista_de_ Negocios_\&_PYMES_V2_N4_1.pdf

Martínez, J. (2005). El sector informal en México. El Cotidiano, (130), 31-45. https://www.redalyc.org/pdf/325/32513005.pdf

Meza, E. (11 de septiembre de 2020). Gobierno aumentará 20\% el apoyo a MIPyMES y dará prioridad a créditos en el 2021. El Economista. Recuperado el 22 de diciembre de 2020, de https://www.eleconomista.com.mx/empresas/Gobierno-aumentara-20-el-apoyo-a-MIPyMESy-dara-prioridad-a-creditos-en-2021-20200911-0061.html

Moreno, T. (1 de julio de 2009). México reclasifica a las Pymes. Expansión. Recuperado el 17 de 
diciembre de 2020, de https://expansion.mx/emprendedores/2009/06/30/el-gobiernoreclasifica-las-pymes

Organización para la Cooperación y el Desarrollo Económicos, (2018). Financiamiento de PyMEs y emprendedores 2018: Un marcador de la OCDE. OCDE. https://www.oecd.org/cfe/smes/SPA-Highlights-Financing-SMEs-and-Entrepreneurs2018.pdf

Rodríguez, E., y Saldívar, B. (16 de julio de 2020). La esperanza de los negocios en México. El Economista. Recuperado el 17 de diciembre de 2020, de https://www.eleconomista.com.mx/empresas/La-esperanza-de-vida-de-los-negocios-enMexico-es-de-7.8-anos-Inegi-20200716-0148.html

Rojas, L. (2017). Situación del financiamiento a PyMEs y empresas en América Latina. Banca de Desarrollo de América Latina-Corporación de Estudios para Latinoamérica. https://scioteca.caf.com/bitstream/handle/123456789/1076/FINANCIAMIENTO\%20A\%20P YMES\%20y\%20EMPRESAS\%20NUEVAS\%20EN\%20AL-300617.pdf

Romero, I. (2006). La Pyme en la economía global. Hacia una estrategia de fomento empresarial. Problemas del Desarrollo, 37(146), 31-50. https://www.researchgate.net/publication/327129333_Las_PYME_en_la_economia_global_ Hacia una estrategia de fomento empresarial

Universidad Nacional Autónoma de México. (2015). Capítulo I. Las PYMES en México. UNAM. http://www.ptolomeo.unam.mx:8080/xmlui/bitstream/handle/132.248.52.100/89/A4.pdf

Vecino, F., y Boggio, J. (2006). Reflexiones sobre las políticas de fomento y apoyo a micros, pequeñas y grandes empresas en México y las experiencias en países de la OCDE [ponencia]. $\mathrm{X}$ Congreso Anual de la Academia de Ciencias Administrativas, A.C., San Luis Potosí, México. Recuperado el 16 de octubre de 2020, de http://acacia.org.mx/busqueda/pdf/P26T17.pdf

Verbano, K., \& Venturini, C. (2013). Managing risks in SMEs: A literature review and research agenda. Journal of Technology Management \& Innovation, 8(3), 186-197. https://www.researchgate.net/publication/260770823_Managing_Risks_in_SMEs_A_Literat ure_Review_and_Research_Agenda 